\title{
Gemeinschaft selbst bauen
}

\section{Eine Studie über neue Wohnkonzepte jenseits des Standards}

\author{
Nicola Hilti · Eva Lingg
}

Eingegangen: 31. Mai 2021 / Angenommen: 23. Juni 2021 / Online publiziert: 11. November 2021

(C) Der/die Autor(en) 2021

Zusammenfassung Das hier vorgestellte angewandte Forschungs- und Entwicklungsprojekt „,Wohnen im Rohbau“, ,Hallenwohnen“ und ,Selbstausbauloft ${ }^{\text {- }}$ - neue Wohnkonzepte zwischen Gemeinschaft und Selbstbau“ ist am Institut für Soziale Arbeit und Räume der OST - Ostschweizer Fachhochschule (Standort St. Gallen) angesiedelt und befasst sich mit drei Wohnkonzepten, die derzeit in der Schweiz als neuste und vielleicht auch radikalste gelten können: dem „Wohnen im Rohbau“, dem „Hallenwohnen“ und dem „Selbstausbauloft“, allesamt von Wohnbaugenossenschaften getragen. Diese drei genannten Konzepte werden mittels verschiedener qualitativer Methoden untersucht. Ziel der Studie ist es, praxisrelevante Erkenntnisse über Herausforderungen und Gelingensbedingungen bezogen auf 1) die Projektentwicklung und -planung, 2) die Projektumsetzung sowie 3) die Aneignung durch die Bewohnerinnen und Bewohner im Wohnen und deren Zusammenleben zu gewinnen und für künftige ähnliche Projekte nutzbar zu machen. Übergeordnet gilt es, einen Beitrag zur Diversifizierung und Bedürfnisorientierung des schweizerischen Wohnungsmarktes zu leisten. Denn dieser zeichnet sich nach wie vor durch eine relativ hohe Standardisierung aus - bei gleichzeitig zunehmender Pluralisierung und Ausdifferenzierung von Wohnbedürfnissen und Haushaltsformen.

Schlüsselwörter Sozialer Wandel · Wohnen · Neue Wohnkonzepte ·

Schweizerischer Wohnungsmarkt · Selbstbau

Prof. Dr. Nicola Hilti $(\bowtie) \cdot$ Prof. Dr. Eva Lingg

Institut für Soziale Arbeit und Räume, OST - Ostschweizer Fachhochschule,

Rosenbergstrasse 59, 9001 St. Gallen, Schweiz

E-Mail: nicola.hilti@ost.ch

Prof. Dr. Eva Lingg

E-Mail: eva.lingg@ost.ch 


\section{Building community yourself}

A study of new housing concepts beyond the standard

Abstract The applied research project presented here "'Wohnen im Rohbau', 'Hallenwohnen' und 'Selbstausbauloft'—neue Wohnkonzepte in der Schweiz" ('living in unfinished buildings', 'living in industrial buildings' and 'do-it-yourself lofts' - new housing concepts in Switzerland) is affiliated with the Institute for Social Work and Spaces of the OST - Ostschweizer Fachhochschule (location St. Gallen) and deals with three housing concepts that can currently be considered the newest and perhaps most radical in Switzerland: 'Wohnen im Rohbau', 'Hallenwohnen' und 'Selbstausbauloft' - all of which are supported by housing cooperatives. These three concepts are investigated by means of different qualitative methods. The aim of the study is to gain practice-relevant insights into challenges and conditions for success related to (1) project development and planning, (2) project implementation, and (3) practices of everyday life of residents and their coexistence, and to make these useful for future projects that are similar. The overriding goal is to contribute to the diversification and needs orientation of the Swiss housing market. This is because the market is still characterized by a relatively high degree of standardization-while at the same time the pluralization and differentiation of housing needs and household forms is increasing.

Keywords Social change $\cdot$ Housing $\cdot$ New housing concepts $\cdot$ Swiss housing market · Do-it-yourself building construction

\section{Ausgangslage: Wohnformen jenseits des Standards und im Kontext des sozialen Wandels}

„Wohnen im Rohbau“, „Hallenwohnen“ und „Selbstausbauloft“: Hinter diesen Bezeichnungen stecken die neusten und vielleicht radikalsten Wohnkonzepte, die in der Schweiz derzeit realisiert werden. Trägerschaften derselben sind experimentierfreudige Wohnbaugenossenschaften. In diesen Konzepten nehmen sie Wohnbedürfnisse eines Teils der Bevölkerung auf, welche sowohl Ansprüche an Selbstbau als auch an Gemeinschaft im Wohnen beinhalten. Selbstbau meint hier die Möglichkeit, eine Wohneinheit im Rohbau, die von der Trägerschaft zur Verfügung gestellt wird, nach persönlichen Vorstellungen als Einzelperson oder gemeinsam als Gruppe auszubauen. Gemeinschaft bezieht sich neben dem gemeinsamen Ausbauen weiter auf den Wunsch, mit anderen innerhalb einer Wohneinheit gemeinschaftlich zusammenzuleben, und dies aus einer Grundhaltung heraus (nicht z.B. als reines Mittel zum Zweck). ${ }^{1}$

Die genannten drei Konzepte enthalten jeweils Elemente des Selbstbaus sowie des gemeinschaftlichen Wohnens, jedoch in je unterschiedlicher Ausprägung: So

\footnotetext{
1 Diese neuen Wohnformen zwischen Selbstbau und Gemeinschaft fanden sich bisher teilweise im gesetzlichen Graubereich wieder, indem verlassene Industrieareale wie das Zürcher Labitzke-Areal um die Jahrtausendwende von Besetzergruppen und Mieterkollektiven umgenutzt worden sind.
} 
steht beim Konzept „Wohnen im Rohbau“ der Genossenschaft Kraftwerk1 in Zürich der Selbstbau im Vordergrund, wohingegen bei den Konzepten „Hallenwohnen“ der Genossenschaft Kalkbreite in Zürich sowie „Selbstausbauloft“ der Genossenschaft Warmbächli in Bern das Selbermachen Hand in Hand geht mit dem Ziel, gemeinschaftlich zu wohnen (und vielfach auch zu arbeiten). Beim „Wohnen im Rohbau“ stellt die Genossenschaft als Trägerschaft konventionell geschnittene Wohnungen zur Verfügung, die die Bewohnerinnen und Bewohner eigenhändig nach ihren Vorstellungen ausbauen können. Beim „Hallenwohnen“ und beim „Selbstausbauloft“ wird einer (oder mehreren) Gruppen von circa zehn bis 20 Personen je eine Halle im Rohbau zur Verfügung gestellt, welche sie nach eigenen Bedürfnissen weitgehend frei gestalten und ausbauen können (Breit und Gürtler 2018, S. 25; Schumacher 2021, i.E.); auf diese Halle müssen sich die Interessentinnen und Interessenten als Gruppe bewerben. Dabei werden individuelle Wohnflächen zugunsten gemeinschaftlicher Wohnflächen maximal reduziert. Anstelle konventioneller Raumaufteilungen und Grundrisse treten offene, kollektiv organisierte Flächen (Simon 2019, S. 34). Einzige Limitierungen sind baurechtliche Vorgaben sowie finanzielle Ressourcen. Wie die Menschen dort zukünftig leben wollen, entscheiden sie schon in partizipativen Entwicklungsprozessen maßgeblich mit.

Diese Trends zu Gemeinschaft und Selbstbau im Wohnen sind eine Reaktion auf die fortschreitende Individualisierung und damit verbundene vermehrte Wahlfreiheiten in Bezug auf die Gestaltung des Lebens und Wohnens (Schmid 2019, S. 208). Einerseits sind Menschen heute offener für verschiedene Lebens- und Wohnformen, andererseits sind sie aber auch vermehrt auf sich allein gestellt und verlieren traditionelle Bindungen und Sicherheiten (Beck 1986; Reckwitz 2017; Breit und Gürtler 2018). Dies kann Vereinsamung, Überforderung oder Überlastung und ein stärkeres Bedürfnis nach Gemeinschaft auslösen (Breit und Gürtler 2018), sodass auch das Interesse an neuen Wohnformen wächst. ${ }^{2}$

Neue Wohnkonzepte wie „Wohnen im Rohbau“, „Hallenwohnen“ und „Selbstausbauloft" ermöglichen den Bewohnerinnen und Bewohnern, in der physisch-materiellen und sozialen Ausgestaltung ihres Wohnens flexibel auf sich wandelnde Bedürfnisse einzugehen (Schmid 2019; Breit und Gürtler 2018). Den Trägerschaften erlauben derlei Konzepte eine kurzfristigere Reaktion auf sich zunehmend schneller verändernde Nutzungsbedürfnisse (Breit und Gürtler 2018, S. 2), Haushaltsstrukturen oder Arbeitsmodelle (Zemp et al. 2018, S. 118) und eine dichtere Nutzung der Flächen (Schneider o.J., S. 4).

Zugleich knüpfen die Trägerschaften ebenso wie die Bewohnerinnen und Bewohner, aber auch andere Akteurinnen und Akteure des Wohnungsmarktes (z. B. aus der Politik und Verwaltung) vielfältige Erwartungen an solche neuen Wohnkonzepte, was ihre sozialintegrative Kraft für die Bewohnerschaft, die Nachbarschaft beziehungsweise das Quartier betrifft. Dementsprechend erachten sie diese Konzepte nicht

\footnotetext{
2 Dieses Interesse ist zuletzt auch in der Politik erwacht: Eine parlamentarische Anfrage einer sozialdemokratischen Nationalrätin vom 18. März 2021, in der auch explizit das Konzept des Hallenwohnens erwähnt wird, lautet: „Neue Wohnformen. Was unternimmt der Bund?““ (https:/www.parlament.ch/de/ratsbetrieb/ suche-curia-vista/geschaeft?AffairId=20213313) Der Schweizerische Bundesrat ist aufgefordert, die Bedeutung neuer Wohnformen einzuschätzen, und zu den Auswirkungen neuer Wohnformen auf den gesellschaftlichen Zusammenhalt und den Wohnflächenverbrauch Stellung zu nehmen.
} 
selten auch als probate Ansatzpunkte, um gesellschaftliche Bruchlinien zu überwinden, indem nachbarschaftliche Hilfe- und Unterstützungssysteme geschaffen oder nahräumliche Netzwerke gestärkt werden, oder indem allgemeiner der soziale $\mathrm{Zu}$ sammenhalt respektive „Kitt“ unter den Bewohnerinnen und Bewohnern bestimmter Gebiete (wieder-)hergestellt wird (Reutlinger et al. 2015; Hilti und Lingg 2021).

Die Akteurinnen und Akteure, welche neue Wohnkonzepte wie die beschriebenen realisieren, bemühen sich häufig in besonderer Weise darum, keine sozial homogenen, isolierten „Inseln der Seligen“ zu sein, sondern ein selbstverständlicher Teil einer diversen, integrativen Stadtgesellschaft. Dies wird versucht, indem beispielsweise die Bewohnerzusammensetzung stark gesteuert und vielfältige öffentliche Nutzungen offeriert werden.

Während es zu verschiedenen Formen des gemeinschaftlichen Wohnens bereits etliche Untersuchungen gibt (z.B. Bundesinstitut für Bau-, Stadt- und Raumforschung 2020; Schmid 2019; Spellerberg 2018; Emmenegger et al. 2017; Philippsen 2014; Wonneberger 2015; Fedrowitz und Gailing 2003), sind die neusten Wohnkonzepte „Wohnen im Rohbau“, „Hallenwohnen“ und „Selbstausbauloft“ bislang unerforscht.

\section{Forschungsdesign: Lernen von den Pionierinnen und Pionieren}

Bei dieser Lücke setzt das im Januar 2022 startende und 36 Monate laufende angewandte Forschungsprojekt ,"Wohnen im Rohbau', ,Hallenwohnen“ und ,Selbstausbauloft " - neue Wohnkonzepte in der Schweiz" des Instituts für Soziale Arbeit und Räume der OST - Ostschweizer Fachhochschule an. Es befasst sich mit den genannten drei Wohnkonzepten. Die drei Konzepte sind in unterschiedlichen Entwicklungsstadien: Während die Hallenbewohnerinnen und -bewohner vor Kurzem eingezogen sind, läuft beim „Wohnen im Rohbau“ und beim „Selbstausbauloft“ der Findungs- respektive Selbstorganisationsprozess der künftigen Bewohnerinnen und Bewohner; der geplante Bezug ist auf ca. 2023/2024 datiert.

Mit der Untersuchung der drei skizzierten Wohnkonzepte soll ein Beitrag zur erfolgreichen Umsetzung künftiger ähnlicher Konzepte geleistet werden - und übergeordnet zu einem insgesamt diverseren und bedürfnisorientieren Wohnungsangebot, als es heute in der Schweiz besteht. Hierfür werden die Erfahrungen der „Pionierinnen und Pioniere“ - hier: der Vertreterinnen und Vertreter der Wohnbaugenossenschaften und der (künftigen) Bewohnerinnen und Bewohner - erhoben und systematisch aufgearbeitet, und zwar in Bezug auf: 1) die Projektentwicklung und -planung (z.B. den involvierten Akteurinnen und Akteuren, der Prozessgestaltung), ggf. auch der Finanzierung und der Rechtsform; 2) die bauliche Umsetzung (z. B. die Gestaltung von Grundrissen, der Einsatz von Materialitäten); 3) die Aneignung im Sinne der eigentätigen Auseinandersetzung mit der vorgefundenen materiellen und sozialen Welt (Stichworte: Nutzungsweisen, Umgang mit dem Gebauten, Zusammenleben, Bedeutungen des Wohnens, Verhältnis von Privatheit und Öffentlichkeit etc.).

Methodisch im Mittelpunkt stehen einerseits Interviews mit (zukünftigen) Bewohnerinnen und Bewohnern (zu verschiedenen Zeitpunkten) u. a. über ihre Beweg- 
gründe, Erwartungen und Erfahrungen bezogen auf die genannten Wohnkonzepte; hinzu kommen Begehungen und teilnehmende Beobachtungen (z. B. Bewohnerversammlungen, Planungssitzungen der Bewohnerinnen und Bewohner). Andererseits werden Interviews mit Genossenschaftsvertreterinnen und -vertretern sowie weiteren relevanten Akteurinnen und Akteuren (z. B. Planungsfachleuten) geführt. Wichtiges Kontextmaterial bilden relevante Dokumente zum „Wohnen im Rohbau“, „Hallenwohnen“ und „Selbstausbauloft" wie beispielsweise Protokolle, Zeitungsberichte, Filmmaterial u.a.m.

Die so erhobenen Daten werden inhaltsanalytisch (Mayring 2010) und punktuell sinnrekonstruierend ausgewertet mit dem Ziel, konkrete praxisrelevante Aussagen über Herausforderungen und Gelingensbedingungen bei der Entwicklung, Planung und Umsetzung der untersuchten Konzepte herauszuarbeiten. Diese Aussagen werden weiter auf ihre Übertragbarkeit auf andere ähnliche Konzepte hin geprüft. All dies gilt es schließlich in geeigneter Form aufzubereiten und den relevanten Akteurinnen und Akteuren aus Wohnungswirtschaft, Politik, Verwaltung sowie der interessierten Öffentlichkeit zugänglich zu machen.

Funding Open access funding provided by University of Applied Sciences Eastern Switzerland

Open Access Dieser Artikel wird unter der Creative Commons Namensnennung 4.0 International Lizenz veröffentlicht, welche die Nutzung, Vervielfältigung, Bearbeitung, Verbreitung und Wiedergabe in jeglichem Medium und Format erlaubt, sofern Sie den/die ursprünglichen Autor(en) und die Quelle ordnungsgemäß nennen, einen Link zur Creative Commons Lizenz beifügen und angeben, ob Änderungen vorgenommen wurden.

Die in diesem Artikel enthaltenen Bilder und sonstiges Drittmaterial unterliegen ebenfalls der genannten Creative Commons Lizenz, sofern sich aus der Abbildungslegende nichts anderes ergibt. Sofern das betreffende Material nicht unter der genannten Creative Commons Lizenz steht und die betreffende Handlung nicht nach gesetzlichen Vorschriften erlaubt ist, ist für die oben aufgeführten Weiterverwendungen des Materials die Einwilligung des jeweiligen Rechteinhabers einzuholen.

Weitere Details zur Lizenz entnehmen Sie bitte der Lizenzinformation auf http://creativecommons.org/ licenses/by/4.0/deed.de.

\section{Literatur}

Beck, U. (1986). Risikogesellschaft. Auf dem Weg in eine andere Moderne. Frankfurt am Main: Suhrkamp. Breit, S., \& Gürtler, D. (2018). Microliving. Urbanes Wohnen im 21. Jahrhundert. Rüschlikon: GDI Gottlieb Duttweiler Institute.

Bundesinstitut für Bau-, Stadt- und Raumforschung (2020). Cluster-Wohnungen. Eine neue Wohnungstypologie für eine anpassungsfähige Stadtentwicklung. Zukunft Bauen. Forschung für die Praxis, Band 22. https://www.bbsr.bund.de/BBSR/DE/veroeffentlichungen/zukunft-bauen-fp/2020/band-22.html. Zugegriffen: 10. Nov. 2021.

Emmenegger, B., Fanghänel, I., \& Müller, M. (2017). Nachbarschaften in genossenschaftlichen Wohnsiedlungen. Als Zusammenspiel von gelebtem Alltag, genossenschaftlichen Strukturen und gebautem Umfeld - Ein Beitrag zur sozialen Nachhaltigkeit. Luzern: Hochschule Luzern.

Fedrowitz, M., \& Gailing, L. (2003). Zusammen wohnen. Gemeinschaftliche Wohnprojekte als Strategie sozialer und ökologischer Stadtentwicklung. Dortmunder Beiträge zur Raumplanung,, Bd. 112. Dortmund: IRPUD.

Hilti, N., \& Lingg, E. (2021). Nachbarschaft. In F. Kessl \& C. Reutlinger (Hrsg.), Sozialraum - eine elementare Einführung. Wiesbaden: VS. 
Mayring, P. (2010). Qualitative Inhaltsanalyse. Grundlagen und Techniken (11. Aufl.). Weinheim: Beltz. http://www.content-select.com/index.php?id=bib_view\&ean=9783407291424. [Zugegriffen am 30. Mai 2021]

Philippsen, C. (2014). Soziale Netzwerke in gemeinschaftlichen Wohnprojekten. Eine empirische Analyse von Freundschaften und sozialer Unterstützung. Opladen: Barbara Budrich.

Reckwitz, A. (2017). Die Gesellschaft der Singularitäten. Zum Strukturwandel der Moderne. Frankfurt a. M.: Suhrkamp.

Reutlinger, C., Stiehler, S., \& Lingg, E. (2015). Soziale Nachbarschaften. Geschichten, Grundlagen, Perspektiven. Wiesbaden: Springer VS.

Schmid, S. (2019). Eine Geschichte des gemeinschaftlichen Wohnens. Modelle des urbanen Zusammenlebens (1. Aufl.). Basel: Birkhäuser.

Schneider, N. Hallenwohnen: Chancen und Tücken bei der Vermietung von Wohnraum im Edelrohbau, S. 1-5. https://www.wbg-100jahre.ch/projekte/jubilaeumswettbewerb/einreichungen-wettbewerbsbei trag/. Zugegriffen: 30. Mai 2021.

Schumacher, C. (2021). Sorgetragen im Wohnen - eine Erweiterung. In C. Hannemann, N. Hilti \& C. Reutlinger (Hrsg.), Wohnen heute - zwölf Schlüsselthemen sozialräumlicher Wohnforschung. Stuttgart: Fraunhofer Verlag.

Simon, A. (2019). Gezähmte Wohnträume. In: Hochparterre 19 (6-7). https://www.bsarch.ch/images/ content/medien/2019_06_Hochparterre_Hallenwohnen/Hochparterre_34-39_Hallenwohnen_6-7_ 19.pdf. Zugegriffen: 30. Mai 2021.

Spellerberg, A. (2018). Neue Wohnformen - gemeinschaftlich und genossenschaftlich. Erfolgsfaktoren im Entstehungsprozess gemeinschaftlichen Wohnens. Wiesbaden: Springer VS.

Wonneberger, E. (2015). Neue Wohnformen. Neue Lust am Gemeinsinn? (2. Aufl.). Edition Centaurus Beiträge zur gesellschaftswissenschaftlichen Forschung. Wiesbaden: Springer VS.

Zemp, R., Juppien, A., Jacobi, E. K., Winterberger, F., \& Schwehr, P. (2018). Innovative Wohnformen. Kontext, Typologien und Konsequenzen. Hg. von Wohnbaugenossenschaften Schweiz - Regionalverband Zürich. Hochschule Luzern - Technik \& Architektur. Horw. https://www.hslu.ch/dech/hochschule-luzern/forschung/projekte/detail/?pid=4055\#: :text=Mit\%20dem\%20Projekt\%20 \%22Innovative\%20Wohnformen, wohnpolitische\%20und\%20\%C3\%B6konomische\%20Aspekte \%20dargelegt. Zugegriffen: 30. Mai 2021. 